\title{
Taurine bromamine (TauBr) - its role in immunity and new perspectives for clinical use
}

Janusz Marcinkiewicz

From $17^{\text {th }}$ International Meeting of Taurine

Fort Lauderdale, FL, USA. 14-19 December 2009

\begin{abstract}
This review is an attempt to summarize our knowledge about taurine bromamine (TauBr) properties, its role in innate immunity and its therapeutic potential.

$\mathrm{TauBr}$ and taurine chloramine (TauCl) are major haloamines generated by eosinophils and neutrophils at a site of inflammation. Both haloamines share anti-inflammatory and anti-oxidant properties. TauBr, similarly to TauCl, decreases the production of proinflammatory mediators. Their anti-inflammatory and anti-oxidant activities are enhanced by their ability to induce the expression of heme oxygenase-1 (HO-1). TauCl is more stable than TauBr. On the other hand, only TauBr was found to be highly membrane-permeable showing stronger microbicidal activity than TauCl.

In the light of the anti-inflammatory and antimicrobial properties of TauBr we discuss its therapeutic potential in local treatment of inflammation, especially acne vulgaris, the most common inflammatory skin disorder. TauBr, at non-cytotoxic concentrations, is able to kill Propionibacterium acnes, the skin bacteria involved in pathogenesis of acne vulgaris.

As topical antibiotics used in the therapy of acne are associated with the emergence of resistant bacteria, topical TauBr seems to be a good candidate for an alternative therapy.

Recently, in a double blind trial, the efficacy of TauBr was compared with the efficacy of clindamycin, one of the most common topical antibiotics used in acne therapy. Comparable reduction of acne lesions was observed in the $\mathrm{TauBr}$ and clindamycin groups of patients with mild and moderate inflammatory facial acne vulgaris. We conclude that this pilot study supports our concept that TauBr can be used as a topical agent in the treatment of acne vulgaris, especially in patients who have already developed antibiotic resistance. Further studies are necessary to substantiate the more extended use of $\mathrm{TauBr}$ as an anti-inflammatory and anti-oxidant agent in human medicine.
\end{abstract}

\section{Introduction}

Activated neutrophils and eosinophils generate a variety of reactive oxygen species (ROS). Hypochlorous acid $(\mathrm{HOCl})$ and hypobromous acid $(\mathrm{HOBr})$ are the major reactive oxidants generated by these cells at sites of inflammation. Both agents, components of the human innate immune system, exert strong microbicidal activity, but their excessive production leads to tissue damage [1,2]. Taurine, a sulfur-containing amino acid (2-aminoethane sulphonic acid), is the most abundant

Correspondence: mmmarcin@cyf-kr.edu.pl

Department of Immunology, Jagiellonian University Medical College, Krakow, Poland

Full list of author information is available at the end of the article free amino acid in the leukocyte cytosol $(30 \mathrm{mM})$ and is the major scavenger for both hypohalous acids, $\mathrm{HOCl}$ and $\mathrm{HOBr}$ [3]. The products of the reaction between taurine and $\mathrm{HOBr}$ or $\mathrm{HOCl}$ are taurine bromamine (TauBr, N-bromotaurine) or taurine chloramine ( $\mathrm{TauCl}$, $\mathrm{N}$-chlorotaurine), respectively [4,5].

$\mathrm{TauCl}$ is considered the major haloamine produced in vivo by neutrophils. A large number of reports have shown a key role of $\mathrm{TauCl}$ in innate immunity and suggest its use in therapy of various topical infections as well as chronic inflammatory diseases [6]. In contrast, $\mathrm{TauBr}$ has attracted little attention because the extracellular concentration of bromide is at least 1,000-fold lower than that of chloride [2]. However, brominating 
intermediates such as $\mathrm{HOBr}$ and $\mathrm{TauBr}$ are potent antimicrobial agents in vitro $[7,8]$.

In the present paper we discuss data showing antiinflammatory and microbicidal properties of $\mathrm{TauBr}$ confirming its therapeutic potential. Finally, we demonstrate that $\mathrm{TauBr}$ may be a good candidate for a local treatment of skin inflammatory diseases, especially for acne vulgaris, a hypothesis supported by our recent pilot clinical study [9].

\section{In vivo generation of $\mathrm{TauBr}$}

Neutrophil myeloperoxidase (MPO) and eosinophil peroxidase (EPO) use hydrogen peroxide $\left(\mathrm{H}_{2} \mathrm{O}_{2}\right)$ to oxidize halides and thiocyanate to their respective hypohalous acids. At plasma concentrations of halide $(100 \mathrm{mM}$ chloride; 20-100 $\mu \mathrm{M}$ bromide; $<1 \mu \mathrm{M}$ iodide) eosinophil peroxidase preferentially oxidizes bromide $\left(\mathrm{Br}^{-}\right)$to produce hypobromous acid, $\mathrm{HOBr}$.

$$
\mathrm{Br}^{-}+\mathrm{H}_{2} \mathrm{O}_{2}+\mathrm{H}^{+} \rightarrow \mathrm{HOBr}+\mathrm{H}_{2} \mathrm{O}
$$

Recent studies have shown that $\mathrm{HOBr}$ may also be generated by the MPO-halide system of neutrophils.

$$
\mathrm{HOCl}+\mathrm{Br}^{-} \rightarrow \mathrm{HOBr}+\mathrm{Cl}^{-}
$$

$\mathrm{HOCl}$ and hypochlorite ion $\left(\mathrm{OCl}^{-}\right)$are therefore mainly produced by MPO, while $\mathrm{HOBr}$ and hypobromite $\left(\mathrm{OBr}^{-}\right)$are produced by both EPO and MPO [1,2].

$\mathrm{HOBr}$, at physiological $\mathrm{pH}$, reacts readily with amine compounds to form secondary oxidants such as monobromamines, di-bromamines and amino acid-derived aldehydes. These brominating agents, and $\mathrm{HOBr}$ in particular, contribute to innate immunity by virtue of having the ability to kill micro organisms but they may also damage host tissues and contribute to inflammatory tissue injury $[10,11]$.

As taurine is the most abundant free amino acid in the leukocyte cytosol, TauBr is the major bromamine generated in vivo by eosinophil or neutrophil peroxidase. It is commonly accepted that taurine is the primary scavenger of overproduced or dislocated $\mathrm{HOCl}$ and $\mathrm{HOBr}$. $\mathrm{TauBr}$ and $\mathrm{TauCl}$ are relatively long lived oxidants, and are less toxic than the corresponding hypohalous acids $[4,5,12]$. TauBr is therefore believed to protect cells against damage by $\mathrm{HOBr}[1,2]$. However, in contrast to the large body of evidence demonstrating the role of $\mathrm{TauCl}$ in innate immunity, much less is known about microbicidal properties of TauBr [11-13].

\section{In vitro synthesis and detection of $\mathrm{TauBr}$}

To determine the function of $\mathrm{TauBr}$, taurine mono-bromamine has been synthesized in vitro and used in a variety of experimental systems $[8,10]$. In our studies we have used the following protocol for synthesis and detection of $\mathrm{TauBr}$ in vitro.
TauBr preparation: $\mathrm{HOBr} / \mathrm{OBr}^{-}$was generated by mixing equimolar amounts of $\mathrm{HOCl}^{-}$and $\mathrm{NaBr}^{-}$. Then, the product of this reaction was mixed with taurine. The mono-bromamine was obtained with a 10-fold excess of amine over the amount of $\mathrm{HOBr} / \mathrm{OBr}^{-}$. Each preparation of TauBr was monitored by UV absorption spectra $(\lambda=200-400 \mathrm{~nm})$ to assure the authenticity of monobromamine (TauBr) (Fig. 1). Taurine bromamines $\left(\mathrm{TauBr}, \mathrm{TauBr}_{2}\right)$ have absorption spectra similar to those of chloramines, but shifted $36 \mathrm{~nm}$ towards longer wavelengths. The concentration of synthesized $\mathrm{TauBr}$ (taurine mono-bromamine) was determined using the molar extinction coefficient $415 \mathrm{M}^{-1} \mathrm{~cm}^{-1}$ at $\mathrm{A}_{288}[1,8]$.

\section{$\mathrm{TauBr}$ and immune system}

Our current knowledge concerning the physiological functions of $\mathrm{TauBr}$ is based mainly on experimental models investigating the effects of exogenous $\mathrm{TauBr}$ $[9,10]$. In studies with isolated leukocytes, evidence for the formation of endogenous brominating agents was obtained, but long lived bromamines were not detected in the medium. One may speculate that the reduction of $\mathrm{HOBr}^{-}$and bromamines by $\mathrm{H}_{2} \mathrm{O}_{2}$ ' produced by activated leukocytes, may account for these results. Indeed, in vitro studies from a number of laboratories show that $\mathrm{TauBr}$, similarly to $\mathrm{HOBr} / \mathrm{Br}^{-}$, is reduced by $\mathrm{H}_{2} \mathrm{O}_{2}$, resulting in the loss of oxidizing and brominating activity $[2,10]$.

$\mathrm{TauBr}+\mathrm{H}_{2} \mathrm{O}_{2}=\mathrm{Tau}+\mathrm{H}^{+}+\mathrm{Br}^{-}+\mathrm{O}_{2}$.

The ability of $\mathrm{TauBr}$, to react and inactivate $\mathrm{H}_{2} \mathrm{O}_{2}$ [1] probably contributes to the reported "antioxidant" and anti-inflammatory properties of this compound. In addition, both $\mathrm{TauBr}$ and $\mathrm{TauCl}$ can induce the synthesis of heme oxygenase-1 (HO-1) [14], a stress-inducible enzyme, which also has antioxidant and anti-inflammatory capacity. $\mathrm{TauBr}$, similarly to $\mathrm{TauCl}$, is a powerful regulator of inflammation [10,15-17]. Both taurine

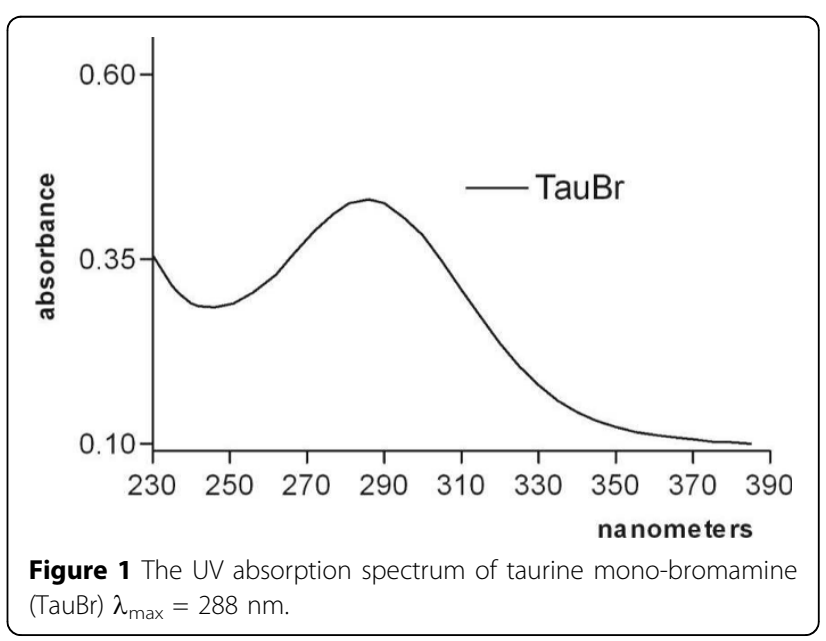


haloamines exert anti-inflammatory properties by suppressing the production of such mediators as nitric oxide, PGE ${ }_{2}$, TNF- $\alpha$, IL-6, IL-8, IL-12 and chemokines in both rodent and human leukocytes $[10,15,18,19]$. Studies investigating the mechanisms of action of $\mathrm{TauCl}$ have shown that it inhibits the activation of NF- $\kappa \mathrm{B}$, a potent signal transducer for inflammatory cytokines, by oxidation of IkB- $\alpha$ at $\mathrm{Met}^{45}$, and recent studies have extended these findings to $\mathrm{TauBr}[16]$.

While the anti-inflammatory properties of endogenous $\mathrm{TauCl}$ and $\mathrm{TauBr}$ are well established, it is less clear whether these compounds also contribute to microbial killing. Our data (Fig. 2) show that $\mathrm{TauBr}$ has strong microbicidal activity comparable to $\mathrm{HOCl}$ at micro molar concentrations, while at these concentrations $\mathrm{TauCl}$ did not kill bacteria [8]. These results are in agreement with the studies of Gaut et al. [17], who found that addition of low concentration of bromide $\left(1 \mu \mathrm{M} \mathrm{Br}^{-}\right)$markedly increased bactericidal activity of the complete myeloperoxidase- $\mathrm{H}_{2} \mathrm{O}_{2}$-halide system. Therefore, one may speculate that physiological variations in $\mathrm{Br}^{-}$concentration may amplify neutrophil bactericidal activity, by driving formation of $\mathrm{HOBr}$ and TauBr.

Importantly, $\mathrm{TauBr}$, at bactericidal concentrations $(<200 \mu \mathrm{M})$ does not exert cytotoxic activity [10]. Moreover, it has been shown that $\mathrm{TauBr}$ at physiological concentrations is able to kill the schistosomula of Schistosoma mansoni confirming its role in the defence against parasites [11]. The contribution of chlorinating and brominating oxidants in pathogen killing and tissue injury will also depend on their interactions with other biologically active agents present at a site of inflammation. For example, TauBr may be neutralised by $\mathrm{H}_{2} \mathrm{O}_{2}$, as mentioned above $[2,10]$.

\section{Therapeutic perspectives for $\mathrm{TauBr}$}

Is $\mathrm{TauBr}$ a good candidate for treatment of skin inflammatory diseases? A number of clinical studies

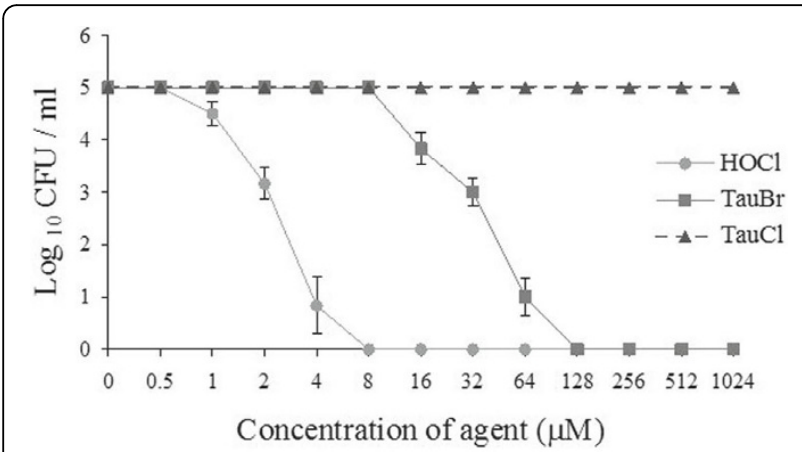

Figure 2 Bactericidal activity of $\mathrm{TauBr}, \mathrm{TauCl}$ and $\mathrm{HOCl}$ against $E$. coli [10]. have shown that $\mathrm{TauCl}$ may be useful for the treatment of various topical infections due to its combination of microbicidal and anti-inflammatory properties. However, in the majority of clinical trials $\mathrm{TauCl}$ was used at very high, non-physiological concentrations $[6,20]$. Much less is known concerning the therapeutic potential of TauBr.

As discussed above, we have shown that $\mathrm{TauBr}$ in vitro has much stronger bactericidal activity than $\mathrm{TauCl}$, with a potency which approaches that of $\mathrm{HOCl}$, the most potent bactericidal agent of MPO-halide system [8]. Interestingly, susceptibility of Propionibacterium acnes (P.acnes) to TauBr appeared to be significantly higher than that of Staphylococcus epidermidis (S.epidermidis) (Fig. 3). Both species belong to the bacterial flora of the skin.

Importantly, $\mathrm{TauBr}$ killed all tested bacteria at noncytotoxic, anti-inflammatory concentrations. From a clinical point of view these data strongly suggest that the therapeutic potential of $\mathrm{TauBr}$ may be similar or even better than that of $\mathrm{TauCl}$.

Based on these studies of the biological properties and functions of taurine haloamines and on our studies demonstrating the selective antimicrobial activity of $\mathrm{TauBr}$, we have examined the clinical efficacy of TauBr in the topical treatment of acne vulgaris, an inflammatory skin disease with bacterial etiology [9].

Acne vulgaris is the most common inflammatory skin disorder in adolescents and young adults [21]. Pathogenesis of acne is complex, involving multiple abnormalities of the pilosebaceous unit, including hyperkeratinisation, sebum production, bacterial proliferation and inflammation. One of the pathogenic factors of acne is $P$. acnes[22,23]. Topical antibacterial agents are an essential element of the armamentarium for the treatment of acne vulgaris $[24,25]$. As $P$. acnes,

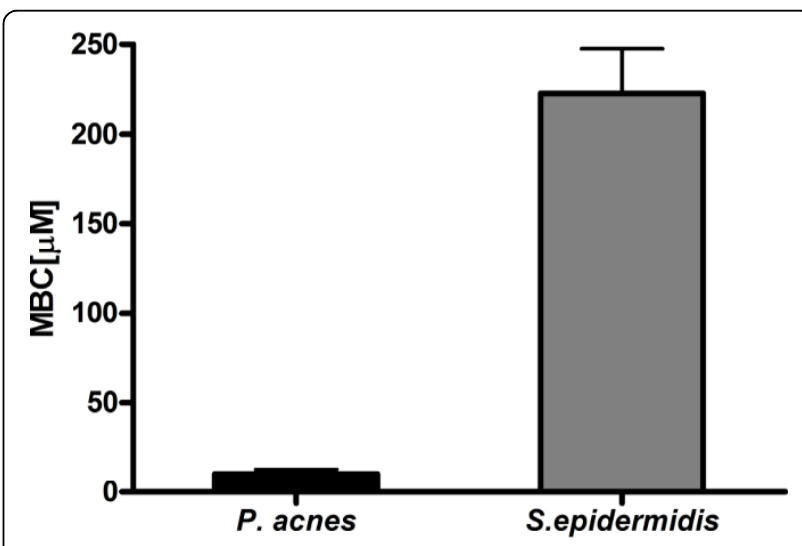

Figure 3 Susceptibility of $P$. acnes and $S$. epidermidis to TauBr. Data are expressed as MBC values (the concentrations of TauBr that cause $100 \%$ inhibition of bacteria growth) [9]. 
a potential pathogenic agent of acne, is extremely sensitive to $\mathrm{TauBr}$, we hypothesized that TauBr may be a good candidate for the topical therapy for acne vulgaris, without the risk of inducing bacterial resistance $[26,27]$.

In a double blind pilot study, the efficacy and safety of TauBr cream was evaluated [9]. Clindamycin gel, one of the most common topical agents in the treatment of acne vulgaris, was used as a control. Forty patients with mild to moderate inflammatory facial acne vulgaris were randomly treated with either $\mathrm{TauBr}$ or clindamycin. After 6 weeks, both treatments produced comparable beneficial results. More than $90 \%$ of patients improved clinically with a similar reduction in number of acne lesions ( 65\%), and with no side effects. Therefore, the results from this clinical pilot are in agreement with previous in vitro data and strongly suggest that $\mathrm{TauBr}$ could be considered a new therapeutic option in inflammatory acne.

\section{Conclusions}

$\mathrm{TauBr}$, the major bromamine generated at the site of inflammation, exerts both anti-inflammatory and antimicrobial properties, at non-cytotoxic concentrations. In addition, its ability to reduce other oxidants (e.g. $\mathrm{H}_{2} \mathrm{O}_{2}$ ) stresses the active role of $\mathrm{TauBr}$ in innate immunity. These biological properties make $\mathrm{TauBr}$ a good candidate for a topical treatment of inflammatory diseases. Further clinical investigations will be required to determine whether $\mathrm{TauBr}$ used in monotherapy or in combination with other agents may be a useful alternative treatment for acne vulgaris.

\section{Abbreviations}

EPO: eosinophil peroxidase; HO-1: heme oxygenase; $\mathrm{HOBr}$ : hypobromous acid; $\mathrm{HOCl}$ : hypochlorous acid; $\mathrm{H} 2 \mathrm{O} 2$ : hydrogen peroxide; IL-6: interleukin -6; IL-8: interleukin-8; IL-12: interleukin-12; Met $^{45}$ : methionine ${ }^{45}$; MPO: neutrophil myeloperoxidase; NF- $\kappa \mathrm{B}$ : nuclear factor- $\kappa \mathrm{B}$; $\mathrm{OBr}^{-}$: hypobromite ion; $\mathrm{OCl}^{-}$: hypochlorite ion; $\mathrm{PGE}_{2}$ : prostaglandin $\mathrm{E}_{2}$ ROS: reactive oxygen species; $\mathrm{TauBr}$ : taurine bromamine, $\mathrm{N}$-bromotaurine; $\mathrm{TauBr}_{2}$ : taurine dibromamine; $\mathrm{TauCl}$ : taurine chloramine, $\mathrm{N}$-chlorotaurine; TNF- $\alpha$ : tumor necrosis factor- $\alpha$.

\footnotetext{
Acknowledgements

I am grateful to Marta Ciszek and Maria Walczewska for the technical assistance.

This paper was partially supported by Jagiellonian University Medical College grant No K/ZBW/000144.

This article has been published as part as part of Journal of Biomedical Science Volume 17 Supplement 1, 2010: Proceedings of the 17th International Meeting of Taurine. The full contents of the supplement are
} available online at http://www.jbiomedsci.com/supplements/17/S1.

\section{Competing interests}

The author declares that he has no competing interests.

Published: 24 August 2010
References

1. Thomas EL, Bozeman PM, Jefferson MM, et al: Oxidation of bromide by the human leukocyte enzymes myeloperoxidase and eosinophil peroxidase. J Biol Chem 1995, 270:2906-2913.

2. Henderson JP, Byun J, Williams MV, et al: Production of brominating intermediates by myeloperoxidase. J Biol Chem 2001, 11:7867-7875.

3. Learn DB, Fried VA, Thomas EL: Taurine and hypotaurine content of human leukocytes. J Leukoc Biol 1990, 48:174-182.

4. Klebanoff SJ: Myeloperoxidase: friend and foe. J Leukoc. Biol. 2005, 77:598-625.

5. Weiss SJ, Klein R, Slivka A, et al: Chlorination of taurine by human neutrophils: evidence for hypochlorous acid generation. J Clin Invest 1982, 70:598-603.

6. Nagl M, Hess MW, Pfaller K, et al: Bactericidal activity of micromolar Nchlorotaurine: evidence for its antimicrobial function in the human defense system. Antimicrob Agents Chemother 2000, 44:2507-2513.

7. Wagner BA, Reszka KJ, McCormick ML, Britigan BE, Evig CB, Burns CP: Role of thiocyanate, bromide and hypobromous acid in hydrogen peroxideinduced apoptosis. Free Radic Res 2004, 38:167-75

8. Marcinkiewicz J, Biedroń R, Białecka A, et al: Susceptibility of Propionibacterium acnes and Staphylococcus epidermidis to killing by MPO- halide system products. Implication for taurine bromamine as a new candidate for topical therapy in treating acne vulgaris. Arch Immunol Ther Exp (Warsz) 2006, 54:61-68.

9. Marcinkiewicz J, Wojas-Pelc A, Walczewska M, Lipko-Godlewska S, Jachowicz R, Maciejewska A, Białecka A, Kasprowicz A: Topical taurine bromamine, a new candidate in the treatment of moderate inflammatory acne vulgaris: a pilot study. Eur J Dermatol 2008, 18:433-9.

10. Marcinkiewicz J, Mak M, Bobek $M$, et al: Is there a role of taurine bromamine in inflammation? Interactive effects with nitrate and hydrogen peroxide. Inflamm Res 2005, 54:42-49.

11. Yazdanbakhsh M, Eckmann CM, Roos D: Killing of schistosomula by taurine chloramine and taurine bromamine. Am J Trop Med Hyg 1987, 37:106-110.

12. Marcinkiewicz J, Chain B, Nowak B, et al: Antimicrobial and cytotoxic activity of hypochlorous acid: interactions with taurine and nitrite. Inflamm Res 2000, 49:280-289.

13. Marcinkiewicz J: Neutrophil chloramines - missing link between innate and acquired immunity. Immunology Today 1997, 18:677-680.

14. Marcinkiewicz J, Grabowska A, Bereta J: Taurine chloramine downregulates the generation of murine neutrophil inflammatory mediators. Immunopharmacology 1998, 40:27-38.

15. Tokunaga $\mathrm{S}$, Kanayama A, Miyamoto $\mathrm{Y}$ : Modification of $\mathrm{IkB} \alpha$ by taurine bromamine inhibits tumor necrosis factor $\alpha$-induced NF-kB activation. Inflamm Res 2007, 56:479-486.

16. Olszanecki R, Marcinkiewicz J: Taurine chloramines and Taurine bromamine induce heme-oxygenase- 1 in resting and LPS-stimulated J774.2 macrophages. Amino Acids 2004, 27:29-35.

17. Gaut JP, Yeh GC, Tran HD, et al: Neutrophils employ the myeloperoxidase system to generate antimicrobial brominating and chlorinating oxidants during sepsis. Proc Natl Acad Sci USA 2001, 98:11961-11966.

18. Kontny E, Chorazy-Massalska M, Rudnicka W, Marcinkiewicz J, Maśliński W: Comparison of taurine chloramine and taurine bromamine effects on rheumatoid arthritis synoviocytes. Amino Acids 2007, 32:447-52.

19. Park E, Schuller-Levis G, Jia JH, et al: Preactivation exposure of RAW 264.7 cells to taurine chloramines attenuates subsequent production of nitric oxide and expression of iNOS mRNA. J Leukoc Biol 1997, 61:161-166.

20. Nagl M, Nguyen VA, Gottardi W, et al: Tolerability and efficacy of Nchlorotaurine in comparison with chloramine $\mathrm{T}$ for treatment of chronic leg ulcers with a purulent coating: a randomized phase II study. $\mathrm{Br} J$ Dermatol 2003, 149:590-597.

21. Burkhart CG, Burkhart CN, Lehmann PF: Acne: a review of immunologic and microbiologic factors. Postgrad Med J 1999, 75:328-331.

22. Jeremy $A H$, Holland $D B$, Roberts $S G$, et al: Inflammatory events are involved in acne lesion initiation. J Invest Dermatol 2003, 121:20-7.

23. Jappe U, Ingham E, Henwood J, et al: Propionibacterium acnes and inflammation in acne; $P$. acnes has T-cell mitogenic activity. $\mathrm{Br} J$ Dermatol 2002, 146:202-9.

24. Dreno B: Topical antibacterial therapy for acne vulgaris. Drugs 2004 64:2389-97. 
25. Guay DR: Topical clindamycin in the management of acne vulgaris. Expert Opin Pharmacother 2007, 8:2625-64.

26. Leyden JJ: Antibiotic resistance in the topical treatment of acne vulgaris. Cutis 2004, 73:6-10.

27. Eady EA, Cove $\mathrm{JH}$, Holland $\mathrm{KT}$, et al: Erythromycin resistant propionibacteria in antibiotic treated acne patients: association with therapeutic failure. Br J Dermatol 1989, 121:51-7.

doi:10.1186/1423-0127-17-S1-S3

Cite this article as: Marcinkiewicz: Taurine bromamine (TauBr) - its role in immunity and new perspectives for clinical use. Journal of Biomedical Science 2010 17(Suppl 1):S3.

Submit your next manuscript to BioMed Central and take full advantage of:

- Convenient online submission

- Thorough peer review

- No space constraints or color figure charges

- Immediate publication on acceptance

- Inclusion in PubMed, CAS, Scopus and Google Scholar

- Research which is freely available for redistribution

Submit your manuscript at www.biomedcentral.com/submit 